\begin{tabular}{|c|c|}
\hline \multirow{3}{*}{ 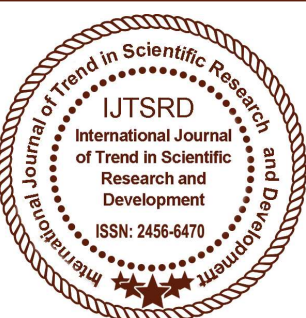 } & $\begin{array}{l}\text { International Journal of Trend in Scientific } \\
\text { Research and Development (IJTSRD) }\end{array}$ \\
\hline & International Open Access Journal \\
\hline & ISSN No: 2456 - 6470 | www.ijtsrd.com | Volume - 2 | Issue -3 \\
\hline
\end{tabular}

\title{
Review and Analysis of WiMAX Technology using different Modulation scheme with AWGN Channel
}

\author{
Arvind Yadav ${ }^{1}$, Er. Praveen Kumar Patidar ${ }^{2}$ \\ M.Tech Student ${ }^{1}$, Professor ${ }^{2}$ \\ Department of Electronics and Communication Engineering, \\ Lakshmi Narain College of Technology, Indore, Madhya Pradesh, India
}

\begin{abstract}
The last few decades, there has been a incredible growth in the wireless communication technology. The growing demand of multimedia services and the growth of Internet related contents lead to increasing interest to high speed communications. The Wireless communication technology, affordable wireless service has become a reality. In wireless communication, radio propagation refers to the behavior of radio waves when they are propagated from transmitter to receiver. In the course of propagation, radio waves are mainly affected by three different modes of physical phenomena: reflection, diffraction, and scattering. In this paper, we analysis of MIMO-OFDM system employing different Modulation scheme is analysed using AWGN channel. The Simulation results show that this is a novel technique for next generation wireless systems using MATLAB toll R2013a.
\end{abstract}

Keywords: $A W G N, M I M O, O F D M, B E R, S N R$

\section{INTRODUCTION:}

The areas of wireless communication have been significantly challenging in the last some years. Over time, several generations have passed to improve the speed and capacity while maintaining an appreciable quality of service [1]. MIMO and OFDM are two combined technology to provide the spectral efficiency and high data rate required for $4 \mathrm{G}$ wireless system technology [1]. The major challenge faced in MIMO-OFDM systems is how to obtain the channel state information accurately and promptly for coherent detection of information symbols. The

communication system bandwidth demand is become higher and higher. In order to transmit data with high speed, the wireless communication having a high spectral efficiency does not directly lead to overcoming the fading. Fading may lead to the reduction of spectral efficiency. It is very difficult to match these requests for the traditional modulation technique, but the MIMO-OFDM system combines the OFDM and MIMO technologies to meet these requirements [2, 3]. MIMO wireless antenna systems have been renowned as a key technology for future wireless communications. The performance of MIMO system can be enhanced by using multiple antennas at transmitting and receiving side to provide spatial diversity [4].

\section{A. WiMAX}

The letters of WiMAX stand for worldwide interoperability for microwave access and it is a technology for point-to-multipoint wireless networking. WiMAX is called the next generation broadband wireless technology which offers high speed, secure and last mile broadband services along with a cellular back-haul and Wi-Fi hotspots. The evolution of WiMAX began a few years ago when scientists and engineers felt the need of having a wireless Internet access and other broadband services which works well everywhere specifically the rural areas or in those areas where it is hard to establish wired infrastructure and economically not feasible. The IEEE 802.16, also known as IEEE WirelessMAN, explored both licensed and unlicensed band of 2-66 GHz which is standard of fixed wireless 
broadband and included mobile broadband application. WiMAX forum, a private organization was formed in June 2001 to coordinate the components and develop the equipment those will be compatible and inter operable. After several years, in 2007, Mobile WiMAX equipment developed with the standard IEEE 802.16e got the certification and they announced to release the product in 2008 , providing mobility access. The IEEE 802.16 e air interface based on Orthogonal Frequency Division Multiple Access (OFDMA) which main aim is to give better performance in non-line-of-sight environments. The mobile WiMAX Fig.1[5], is a technology based on IEEE 802.16 standard [6] developed as a feasible and attractive solution to these problems.

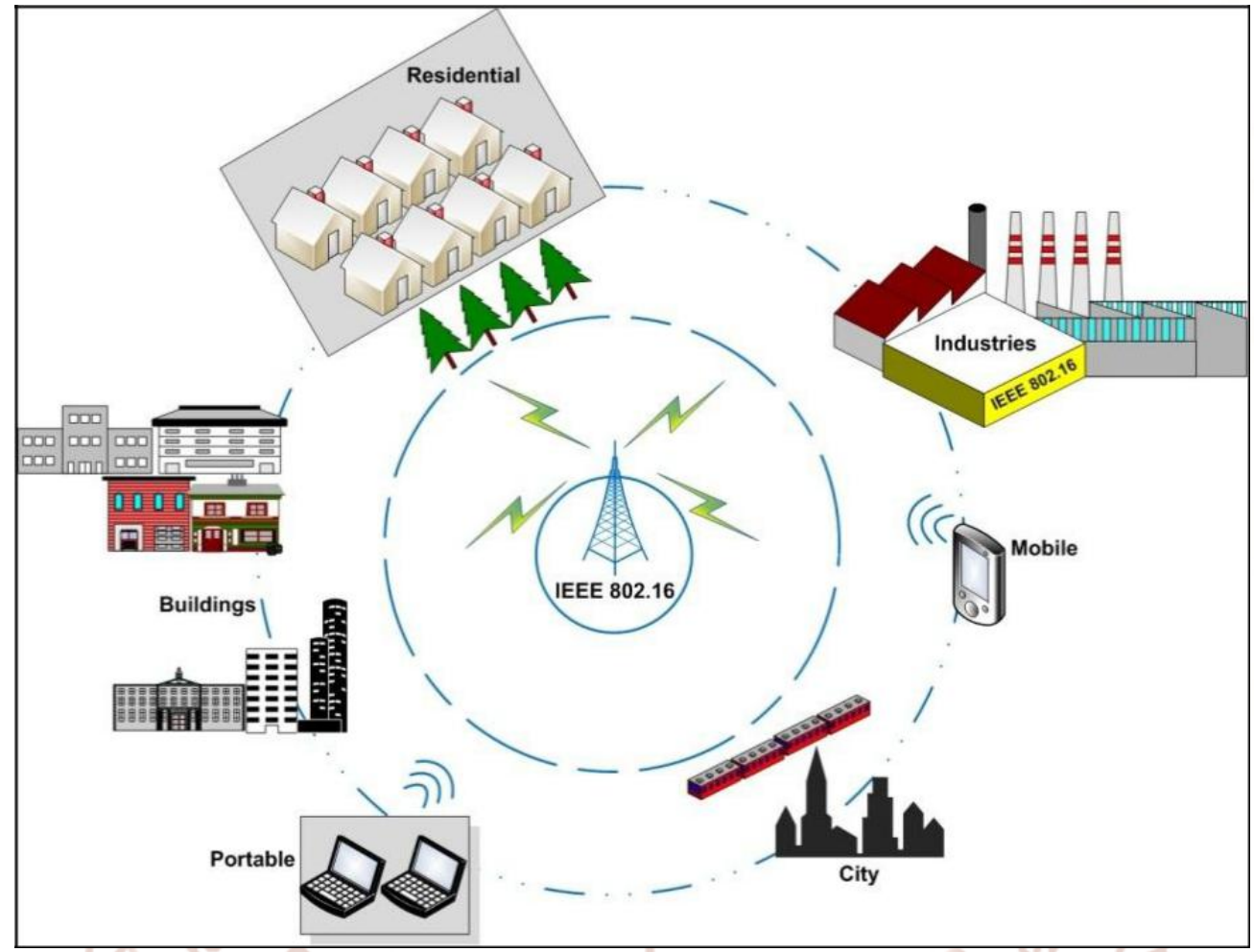

Fig. 1: WiMAX (IEEE 802.16) network architecture

\section{MIMO- OFDM}

A multiple-input

multiple-output

(MIMO) communication system combined with the orthogonal frequency division multiplexing (OFDM) modulation technique can achieve reliable high data rate transmission over broadband wireless channels. OFDM is techniques to digital encode data on the multiple-frequencies. It is widely used in wireless communication networking, Video, audio broadcasting, TV, wireless networks and $4 \mathrm{G}[7,8]$. OFDM plays a major role in both for wireless and wired communications. It started in the 1870 when it was used to carry the information via multiple channels using a telegram [7, 8]. Among many possible technologies for the secondary users' transmission in the spectrum pooling systems, OFDM has already been widely recognized as a particularly novel candidate. This is mainly due to its great flexibility in dynamically allocating the unused spectrum among secondary users as well as its ability to monitor the spectral activities of the licensed users at no extra cost $[11,12,13]$.

OFDM divides the high-rate stream into parallel lower rate data and hence prolongs the symbol duration, thus helping to eliminate Inter Symbol Interference (ISI). It also allows the bandwidth of subcarriers to overlap without Inter Carrier Interference (ICI) as long as the modulated carriers are orthogonal. OFDM therefore is considered as an efficient modulation technique for broadband access in a very dispersive environment. DAB (Digital Audio Broadcasting), High Definition TV, Wireless LANs, Mobile broadband [7]

- IEEE 802.16: It operates in licensed spectrum of $2 \mathrm{Ghz}$ to11 Ghz.

- IEEE 802.20: It operates below $3.5 \mathrm{GHz}$ in the licensed band.

- Up to 155 miles/ h speed $[7,10]$. 
After inserting pilots either to all subcarriers with a specific period of blocks or within a uniform period of frequency bins in all blocks, IDFT block is used to transform the data sequence of length into time domain signal with the following equation 1.

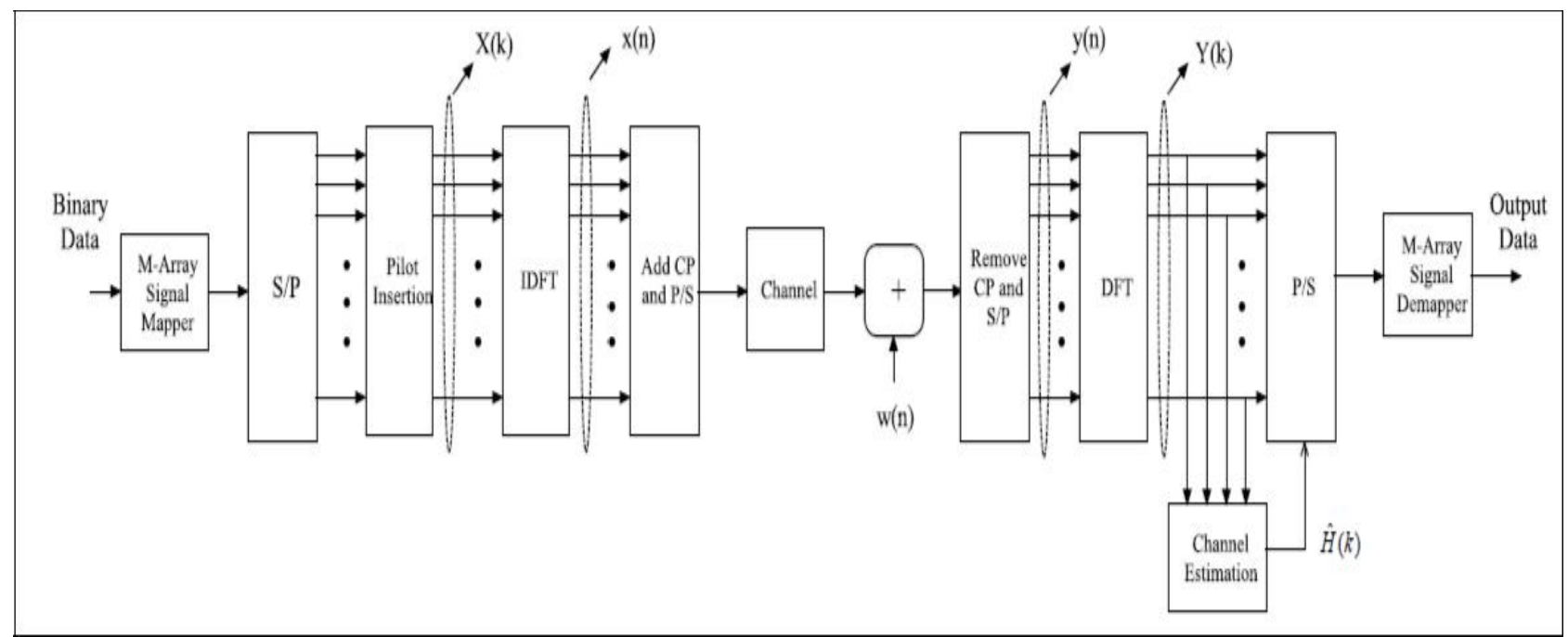

Fig. 2: SISO OFDM System Block Diagram

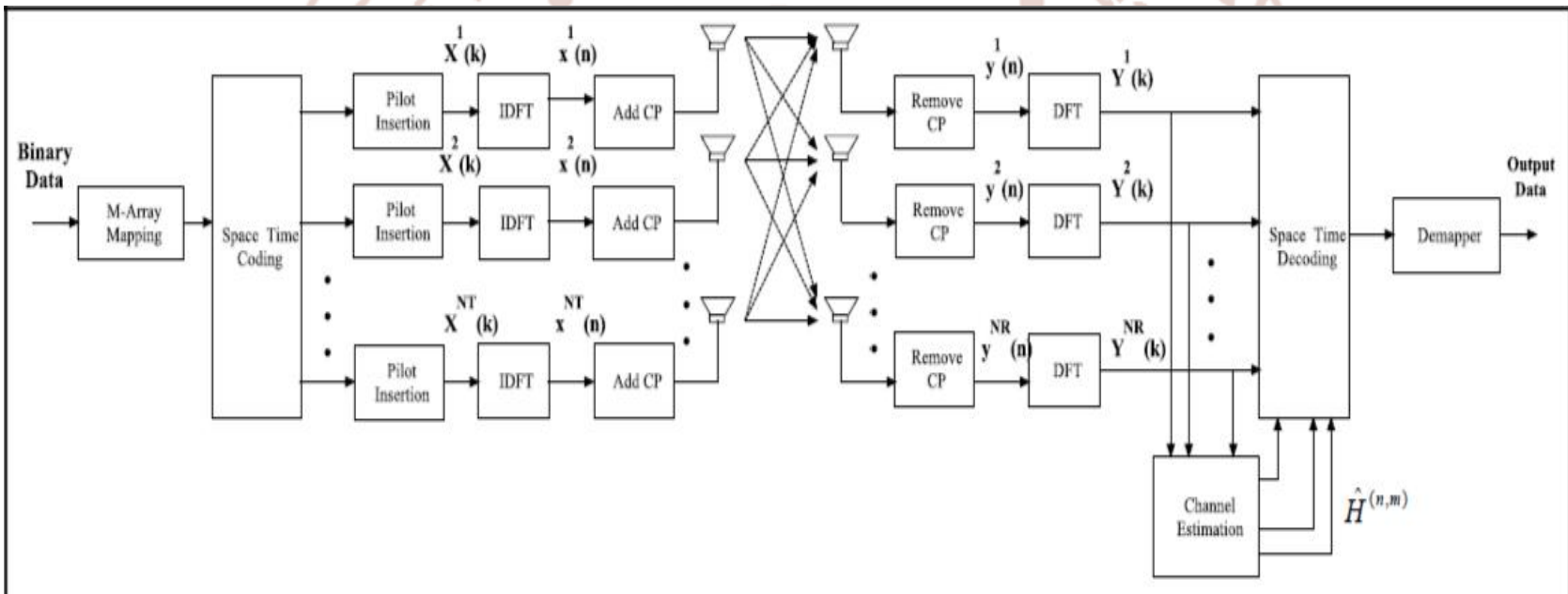

Fig. 3: MIMO OFDM System Block Diagram

Spatial multiplexing Gain: the transmission of multiple data streams over more than one antenna is called spatial multiplexing. The advantage of spatial multiplexing is linear capacity gains in relation to the number of transmit antennas. This gain, referred to as spatial multiplexing gain, is realized by transmitting independent data signals from the individual antennas [14]. Spatial diversity gain: spatial diversity improves the signal quality and achieves a higher signal to noise ratio at the receiver side. Signal power in a wireless channel fluctuates randomly or fades. Diversity is a powerful technique to mitigate fading in wireless links. Consider a space time block coded MIMO-OFDM system [15] equipped with transmit antennas and receive antennas as illustrated in Figure 4. 


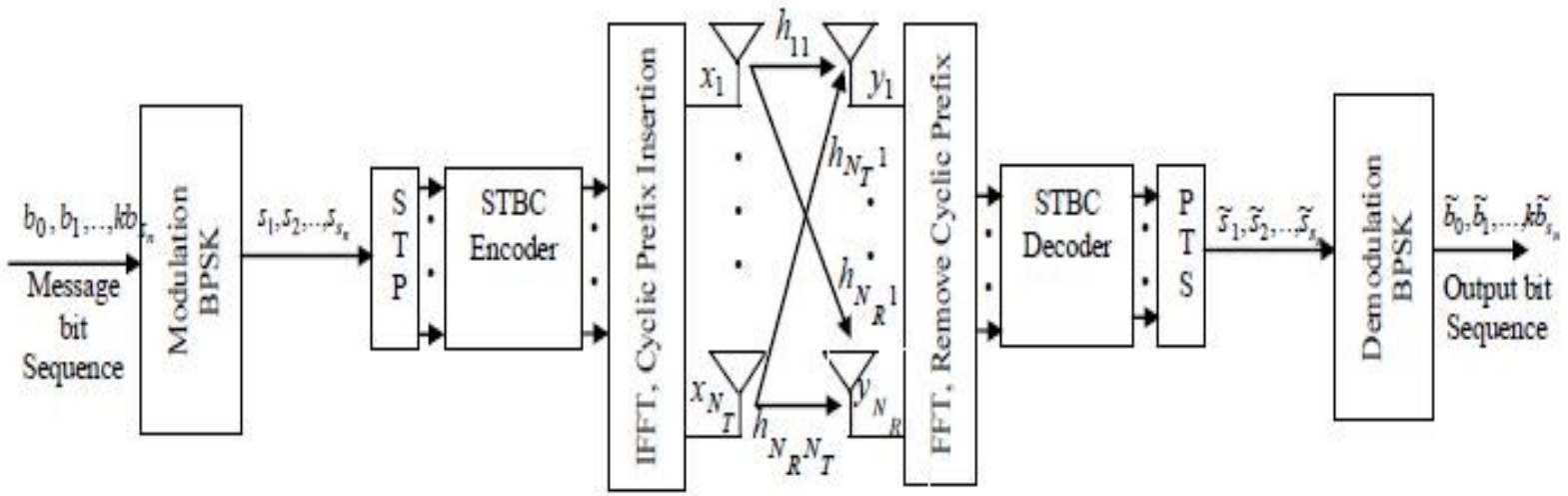

Fig. 4: Block Diagram of Space Time Block Coded MIMO-OFDM system Structure[9]

\section{MODULATION}

Modulation is the process of facilitating the transfer of information over a medium. Sound transmission in air has limited range for the amount of power your lungs can generate. To extend the range your voice reach, we need to transmit it through a medium other than air, such as a phone line or radio. The process of converting information (voice in the case) so that it can be successfully sent through a medium (wire or radio waves) is called modulation.

\section{CHANNEL ESTIMATION}

When a signal containing audio or video is transmitted through the air medium or wireless medium to the receiver $(\mathrm{Rx})$, some of its characteristics are changed due to present noise and interference in the atmosphere[9]. This distorted signal is unable to provide useful information; some of these characteristics are change like frequency, amplitude and Phase. A wideband radio channel is normally time variant and Frequency selective. For an OFDM communication system, the channel transferred at different subcarriers appears in the receiver with unequal frequency and time domains. Therefore, dynamic estimation of the signal is necessary $[7,9]$.

\section{PERFORMANCE ANALYSIS}

To analyze the performance of WiMAX (OFDMsystems) based on the different simulation parameters consider and obtain simulation results. We investigated the BER V/s SNR plot by using AWGN channel. The performance of WiMAX model analysis on used the following parameters as shown in table 1

\section{Table 1: Performance of IEEE 802.16e Parameters}

\begin{tabular}{|l|l|}
\hline \multicolumn{1}{|c|}{ Parameters } & \multicolumn{1}{c|}{ Value } \\
\hline Communication Channel & AWGN \\
\hline Modulation Techniques & $\begin{array}{l}\text { 2-PSK, 4-PSK, 8-QAM } \\
\text { and 16-QAM }\end{array}$ \\
\hline IFFT (Input port size) & 256 \\
\hline CC Code Rate & $1 / 2$ \\
\hline Radio Technology & OFDM \\
\hline Used Scheme & Alamouti \\
\hline $\begin{array}{l}\text { System (Single and } \\
\text { Multiple) tilic }\end{array}$ & MIMO \\
\hline Model & WiMAX 802.16e \\
\hline Calculation Parameters & BER V/s SNR \\
\hline $\begin{array}{l}\text { Simulation-Used } \\
\text { Tool/Software }\end{array}$ & Matlab (R2013a) \\
\hline
\end{tabular}

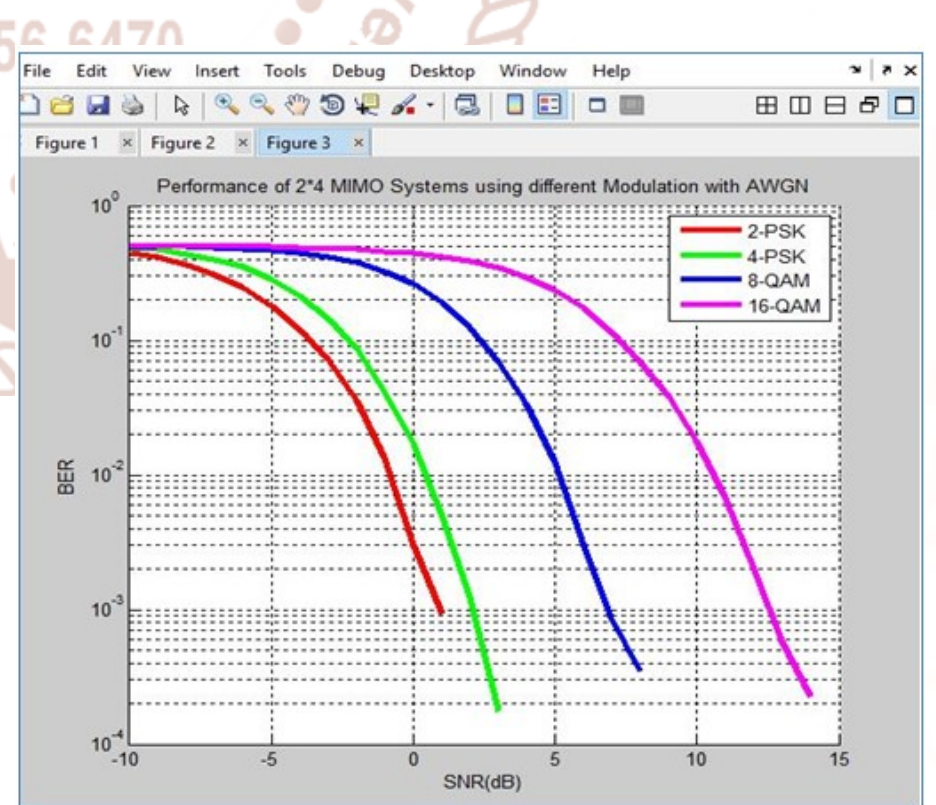

Fig. 5: Performance Analysis of 2*4 MIMO systems using different modulation with AWGN Channel 
International Journal of Trend in Scientific Research and Development (IJTSRD) ISSN: 2456-6470

\section{CONCLUSION}

The WiMAX technology is a broadband wireless data communications technology based around the IEEE 802.16 standard providing high speed data over a wide area. In this performance result is shown in figure 5, we have used the Alamouti scheme with communication AWGN channel and different modulation techniques. The performance is displayed in terms of the BER verses SNR logarithmic plot.

We analysis the 16-QAM, SNR is increased $6.1 \mathrm{~dB}$ on BER at as compared to 8-QAM and Modulation Techniques at a constant signal power.

\section{REFERENCES}

1) Asma Bouhlel, Anis Sakly and Nejib Mansouri "Performance comparison of DFT based MIMO OFDM and FET based MIMO-OFDM" AWICT2015, Elsevier, Procedia Computer Science 73 (2015) 266-273.

2) Ammar Ali Sahrab "MIMO-OFDM: Maximum Diversity Using Maximum Likelihood Detector" 2014 IEEE.

3) Jiang Xuehua, and Chen Peijiang, "Study and Implementation of MIMOOFDM System Based on Matlab," IEEE Computer Society: International Conference on Information Technology and Computer Science, pp. 554-557, 2009.

4) M.Raju "Mean Square Error Analysis in MIMOOFDM System using Pilot based Channel Estimation” 2016 IEEE, (SCOPES-2016).

5) Federal "A Mobile WiMAX Mesh Network with Routing Techniques and Quality of Service Mechanisms" Selected Topics in WiMAX, Chapter 3, INTECH 2013.

6) IEEE 802.16e-2005 Part 16: Air Interface for Fixed and Mobile Broadband Wireless Access Systems Amendment 2: Physical and Medium Access Control Layers for Combined Fixed and Mobile Operation in Licensed Bands, 2005

7) Ramanpreet Kaur, Harjinder Singh "A Review Paper on Performance Analysis of Channel Estimation in MIMO-OFDM System" IJSRD International Journal for Scientific Research \& Development| Vol. 5, Issue 06, 2017 | ISSN (online): $2321-061$.
8) Kala Praveen Bagadi "MIMO-OFDM Channel Estimation using Pilot Carries" International Journal of Computer Applications (0975 - 8887) Volume 2 - No.3, May 2010.

9) Chang-Yi Yang, Yu-Li Chen, Hai-Yan Song "Adaptive Carrier Frequency Offset and Channel Estimation for MIMO-OFDM Systems" 2016 IEEE DOI 10.1109/WAINA.2016.68.

10) While Zhang, Feifei Gao, and Qinye Yin "Blind Channel Estimation for MIMO-OFDM Systems with Low-Order Signal Constellation" IEEE Communications Letters, Vol. 19(3), March 2015.

11) Hamid Shahrokh Shahraki, Kamal MohamedPour "Efficient Power Loading in MIMO-OFDM Based Cognitive Radio Networks" Scientific Research, Communications and Network, 2011, 3, 8-16.

12) R. V. Nee and R. Prasad, "OFDM for Wireless Multimedia Communication," Artech House, London, 2000.

13) U. Berthold, F. Jondral, S. Brandes and M. Schnell, "OFDM-Based Overlay Systems: A Promising Approach for Enhancing Spectral Efficiency [Topics in Radio Communications]," IEEE Communications Magazine, Vol. 45(12), December 2007, pp. 52-58.

14) Kala Praveen Bagadi and Susmita Das "MIMOOFDM Channel Estimation using Pilot Carries" International Journal of Computer Applications (0975 - 8887) Vol. 2 (3), May 2010.

15) Md. Mejbaul Haque, Mohammad Shaifur Rahman and Ki-Doo Kim "Performance Analysis of MIMO-OFDM for $4 \mathrm{G}$ Wireless Systems under Rayleigh Fading Channel" International Journal of Multimedia and Ubiquitous Engineering Vol. 8(1), January, 2013.

16) $\mathrm{Y}$. Chang, R. Zhang "Transmit Optimization for MIMO-OFDM With Delay-Constrained and No-Delay-Constrained Traffic", IEEE Transaction on signal processing, Vol. 54(8), 2006. 